\title{
Self-healing in single and multiple fiber(s) reinforced polymer composites
}

\author{
E. Woldesenbet ${ }^{1}$ \\ ${ }^{1} 2508$ PTH, Mechanical Engineering Department, Baton Rouge, LA 70803 USA
}

\begin{abstract}
You Polymer composites have been attractive medium to introduce the autonomic healing concept into modern day engineering materials. To date, there has been significant research in self-healing polymeric materials including several studies specifically in fiber reinforced polymers. Even though several methods have been suggested in autonomic healing materials, the concept of repair by bleeding of enclosed functional agents has garnered wide attention by the scientific community. A self-healing fiber reinforced polymer composite has been developed. Tensile tests are carried out on specimens that are fabricated by using the following components: hollow and solid glass fibers, healing agent, catalysts, multi-walled carbon nanotubes, and a polymer resin matrix. The test results have demonstrated that single fiber polymer composites and multiple fiber reinforced polymer matrix composites with healing agents and catalysts have provided $90.7 \%$ and $76.55 \%$ restoration of the original tensile strength, respectively. Incorporation of functionalized multi-walled carbon nanotubes in the healing medium of the single fiber polymer composite has provided additional efficiency. Healing is found to be localized, allowing multiple healing in the presence of several cracks.
\end{abstract}

\section{Introduction}

Bones, muscles, wood and other living tissues in animals and plants have developed into highly sophisticated, integrated, hierarchical materials that commonly exhibit multipurpose behavior. Imitation of these naturally existing materials can provide improvement of material design and performance in the future. In addition to achieving structural requirements, bio-inspired selfhealing can also provide an ability to incorporate other functions in engineered materials.

Composite materials have shown tremendous improvements because of advancements made in fibers and resin materials. However, their use is limited due to the difficulty in damage detection and repair as well as lack of extended fatigue and impact resistance. One way to extend their use is through the incorporation of self-healing ability. Healing of materials, such as glass, polymers, and concrete, have been investigated in order to lengthen the service life of these structures [1-4]. In most of these investigations, the healing process involved human intervention and thus the materials were not considered fully self-healed.

Polymer composites have been attractive medium to introduce the autonomic healing concept into modern day engineering materials. To date, there have been significant research in selfhealing polymeric materials [5-10] and several studies specifically in fiber reinforced polymers

This is an Open Access article distributed under the terms of the Creative Commons Attribution-Noncommercial License 3.0, which permits unrestricted use, distribution, and reproduction in any noncommercial medium, provided the original work is properly cited. 
[11-15]. Even though several methods have been suggested in autonomic healing materials, the concept of repair by bleeding of enclosed functional agents has garnered wide attention by the scientific community $[8,11]$.

White et al. [8], Brown et al. [9] and Kessler et al. [16] have successfully demonstrated the development of microencapsulated self-healing polymer composites. Their system employs liquid healing agent microencapsulated in polymeric shells that would fracture under propagating cracks. Once the shells break, the liquid healing agent is released and fills the matrix crack volume. A dispersed catalyst in the matrix polymerizes the released liquid monomer by a chemical process called, ring opening metathesis polymerization (ROMP), thereby completing the self-healing system. The same polymerization methodology has been used in coatingsubstrate design that delivers healing agent to cracks in a polymer coating via a threedimensional microvascular network embedded in the substrate [17]. The researchers have found that crack damage in the epoxy coating has healed repeatedly. Different type of vascular selfhealing approach has also been developed by Williams et al. [18] where self-healing sandwich panels which undergo a compressive stress after impact showed high healing efficiency. However, while giving the extra benefit of autonomic healing, the microchannels within the composite may act as the initial site of damage in the microvascular network composites [17].

In fiber reinforced composites, the introduction of self-healing concept will serve as a means to develop damage tolerant design and repair to damaged parts. In addition to high strength, low specific weight, and impact and corrosion resistance, self-healing fiber reinforced polymer matrix composites can offer the extra benefit of autonomic damage repair. The earliest work in self-healing composites with fibers has been the work by Dry [5] that encompasses glass capsules to contain a liquid resin system that bleed into damage sites upon fracture. Motuku et al. [12] have later suggested the inclusion of tubes in a brittle matrix material for self-repair of cracks at a millimeter length in polymer composites. This concept was developed by considering different critical parameters such as a method of storage and healing agents. In their experiment, they have found that the release of a healing agent through glass is the most suitable method compared to copper and aluminum tubing. However, the first successful use of hollow glass fibers embedded in a composite laminate has been suggested by Bleay et al. [11]. In their work, a repair agent and hardener placed in separate hollow fibers have been consolidated into lamina and then manufactured into composite laminates. The key advantages of the hollow fiber selfhealing concept are that the fibers can be located to match the orientation of the surrounding reinforcing fibers. More recently, several self-healing unidirectional hollow fiber composites have been developed $[13-15,18,19]$. The composite systems showed considerable restoration of mechanical properties such as flexural strength, compressive strength and impact resistance through bleeding of hollow fibers that carry repair materials. Recently, works by Yin et al. [20, 21] have shown self-healing efficiency in epoxy and catalyst/hardener containing microcapsules dispersed in a woven glass fabric reinforced epoxy composites.

Furthermore, studies on the addition of nanoparticles to enhance mechanical properties in polymer composites have shown tremendous progress over the past decade. Lee et al. [22] showed the possibility of using nanoparticles dispersed in polymer films as deposit agents at a damage site in a similar fashion to blood clotting. In their work, the nanoparticles are dispersed in polymer films within a multilayer composite and are studied by integrated computer simulations. Recently, Gupta et al. [23] used fluorescent nanoparticles to show that ligands on the nanoparticles can be selected to help drive nanoparticles into a crack in a microelectronic thin film polymer layer. Verberg et al. [24] modeled the rolling motion of a fluid-driven, particlefilled microcapsule along a heterogeneous, adhesive substrate to determine how the release of the encapsulated nanoparticles can be harnessed to repair damage on the underlying surface. A more recent work by Jeong and Kessler [25] demonstrated over $900 \%$ tensile toughness enhancement when $0.4 \%$ Norbornene-functionalized multiwalled carbon nanotube (MWNT) was incorporated in dicyclopentadiene (DCPD) nanocomposites.

All the different methods in self-healing composites developed so far have their own pros and cons. Despite their advantages, all approaches pose some type of problem with regard to the 
self-healing process. Moreover, most studies have been falling short of showing multiple or repetitive healing in their systems.

The work presented in this study is a major advancement in self-healing composites where catalyst coated hollow fibers are utilized. The first experiment involves a single, catalyst coated hollow fiber that is filled with healing agent and embedded in a resin matrix. The second experiment utilizes functionalized multiwalled carbon nanotubes that are dispersed in the healing agent in order to determine their influence in the healing process. These studies provide the basis for the development of a fiber reinforced polymer composite system that contains commercial solid glass fibers along with the hollow glass fibers. The hollow glass fibers store a monomer healing agent and have a catalyst coated on their outside surface. In the event of damage of the composite, the hollow fibers break and release the healing agent that fills the damage site. This released healing agent polymerizes when it contacts the pre-coated catalyst. Healed, damaged and virgin specimens are tested in tension to demonstrate the self-healing ability of the composites.

\section{Experimental}

\subsection{Materials}

Several materials are combined together to form the self-healing composite specimens. These are: resin matrix composed of epoxy EPON 828 along with an amine based hardener EPI-CURE 3223 from Miller-Stephenson Chemical Co., USA; hollow glass fiber made of borosilicate glass tubing (outside diameter (OD) of $125 \mu \mathrm{m}$ and degree of hollowness of 64\%) from Capillary Tube Supplies Ltd., UK; healing agent - dicyclopentadiene (DCPD) manufactured by Sigma-Aldrich Co., USA; catalyst - first generation Grubb's catalyst by Miller Chemical Co., USA; and sealant - Dow Corning Silastic Rubber 3120RTV by Sigma-Aldrich Co., USA.

In addition to the hollow fibers, solid glass fibers are used to fabricate the integrated fiber reinforced composite specimens. The solid glass fibers are commercial E-glass fibers with a diameter of $12 \mu \mathrm{m}$ and tensile strength of $2750 \mathrm{MPa}$. In the composite where nanoparticles are dispersed in the healing agent, $\mathrm{COOH}$ functionalized multiwalled carbon nanotubes $(\mathrm{COOH}-$ MWNT) from Cheap Tubes Inc., USA are used. Norbornenylethyldimethyl-chlorosilane from Hybrid Plastics Inc., USA, anhydrous pyridine and anhydrous ethylene glycol from SigmaAldrich Co., USA, are also used. Reagents and solvents such as tehrahydrofuran (THF), Chloroform, Dichloromethane (DCM) and acetone are purchased from commercial suppliers and used as received.

\subsection{Specimen Fabrication}

A rubber mold is produced using Dow Corning 3120RTV Silicon Rubber (Red Color) and Dow Corning 1 Catalyst curing agent for the fabrication of test specimens. In this study, three types of self-healing composite specimens are produced. These are, single fiber polymer matrix composite (SFPMC), single fiber polymer matrix composite with functionalized carbon nanotubes (SFPMN) and fiber reinforced polymer composite (FRPMC). The specimen used in the SFPMC and SFPMN has a dimension of $32 \mathrm{~mm} \times 6 \mathrm{~mm} \times 2 \mathrm{~mm}$. The hollow fiber has a length of $42 \mathrm{~mm}$ where $32 \mathrm{~mm}$ is embedded within the resin and $5 \mathrm{~mm}$ on both ends is free.

In the case of SFPMC, the procedure of fabrication starts by filling the hollow glass fiber with a repair material, dicyclopentadiene (DCPD). The DCPD is the same monomer that was used by White et al. [8]. It usually stays in solid form at room temperature and has to be heated up to $110^{\circ} \mathrm{F}$ in order to melt. Since the DCPD has to be in a liquid form all the time during specimen fabrication and testing, chloroform is added at a ratio of $1 \mathrm{ml}$ to $25 \mathrm{ml}$ of DCPD preventing it from solidifying at room temperature. The liquid DCPD mixture is then filled in the hollow fiber by capillary action and sealed at both ends with silicone sealants.

In the case of SFPMN, the procedure followed for norbornene functionalization of the COOH-MWNT is similar to Jeong and Kessler [25]. 1.1g of COOH-MWNT is refluxed in a thionyl chloride with a catalytic amount of $\mathrm{N}, \mathrm{N}^{\prime}$-Dimethylformamide (DMF) $\mathrm{N}_{2}$ at $70{ }^{\circ} \mathrm{C}$ for $48 \mathrm{~h}$. 
The residual $\mathrm{SOCl}_{2}$ is removed by high vacuum distillation giving acyl chloride-functionalized MWNTs (MWNT-COCl). The MWNT-COCl is immediately reacted with $40 \mathrm{~mL}$ of ethylene glycol and $0.1 \mathrm{~mL}$ of pyridine at $120{ }^{\circ} \mathrm{C}$ for $48 \mathrm{~h}$ and then purified. After being cooled, the solution is filtered and rinsed with tehrahydrofuran/THF and acetone. The product is dried under vacuum at $70{ }^{\circ} \mathrm{C}$ for $24 \mathrm{~h}$, giving MWNT-OH. The MWNT-OH is added in 5-norbornene-2yl(ethyl)-chlorodimethylsilane $(25 \mathrm{~mL})$ solution and $0.1 \mathrm{~mL}$ of pyridine and refluxed for $48 \mathrm{~h}$ at $70{ }^{\circ} \mathrm{C}$. The solution is filtered and washed fully with THF and dried under vacuum at $70{ }^{\circ} \mathrm{C}$ for $24 \mathrm{~h}$, giving norbornene-functionalized MWNT.

The product, norbornene-functionalized MWNT, is then mixed with the DCPD mixture (DCPD and chloroform as used in SFPMC) using tip sonicator to fully disperse the functionalized carbon nanotubes in the DCPD mixture, Fig 1. Three different loadings of functionalized MWNT are used, 0.1, 0.3 and $0.5 \mathrm{wt} \%$. The mixture is filled in the hollow glass fiber through capillary action and then sealed at both ends by silicone sealants.

Grubb's catalyst is in a form of powder when it is received and must be set in a liquid form in order to coat the hollow glass fibers thoroughly. Poche and associates [26] found that mixing $10 \mathrm{mg}$ of Grubb's catalyst and $1 \mathrm{ml}$ of dichloromethane (DCM) would enhance the Grubb's catalyst stay in liquid form. They have also found that the Grubb's catalyst is not affected by the DCM. The filled and sealed glass fibers are placed in a dish that contains the Grubb's catalyst and DCM mixture, and rolled in the dish so that they are coated thoroughly on the outside surface. Once the DCM evaporates, the coated glass fibers filled with the healing agent are then placed in the mold to prepare the specimens.

The EPON Resin 828 and EPI-CURE Curing Agent 3223 are mixed together at a ratio of $12: 1$ parts by mass to form the resin system. The mixed resin is poured into the silicone rubber mold. The curing schedule is seven days at room temperature. The final specimen is then demolded from the silicone form and made ready for mechanical testing.

The geometry of the specimen used to demonstrate self-healing in FRPMC is rectangular with dimension of $32 \mathrm{~mm} \times 3 \mathrm{~mm} \times 2 \mathrm{~mm}$. The specimen is a single layer with unidirectional hollow glass fibers and solid glass fibers that are placed in the matrix. Both the hollow glass fiber and the solid glass fibers are entirely embedded in the matrix. The solid fiber is a strand

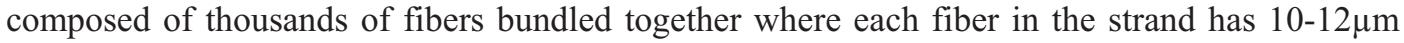
OD.

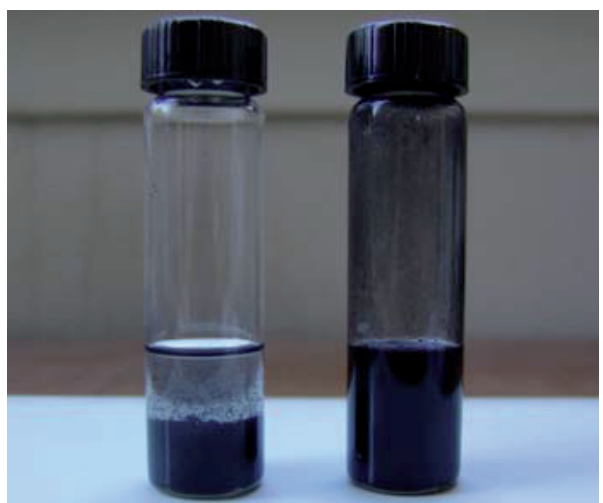

Figure 1. (Left) as supplied MWNTs in a DCPD mixture after one hour and (Right) functionalized MWNT and DCPD mixture after three days 


\section{Testing}

The work presented in this study provides a foundation for the utilization of self-healing of cracks in fully integrated fiber reinforced polymer composites that are well known for their excellent properties such as tensile strength and modulus. Crack is induced by applying flexural load on the composite using a three point bending set-up. A small crack/scratch on the fiber is used to direct the crack when a flexural load is applied on the composite specimen. The purpose of applying a flexural load on the specimen is to break the fiber at a designated location to assist visualization of the healing process. When the fiber breaks, additional cracks are also generated in the matrix within the damage zone. In the case of FRPMC samples, damage is introduced to the solid fibers by actually cutting the solid fibers at their mid-length $(16 \mathrm{~mm})$. Therefore, the damaged solid fibers are two independent fibers that extend from the middle to the end of the specimen in opposite directions. Fabrication of specimen involves damaging the reinforcement fibers in all specimen types except, in the virgin specimen which is fabricated with solid and hollow glass fibers that are not damaged.

The induced cracks in this experiment are transverse to the fiber orientation, and these cracks are expected to grow within the composite and cause catastrophic failure unless the healing process is successful. Similarly, the fracture toughness test performed by Chen et al. [6] and White et al. [8] used tensile load perpendicular to the crack plane to study the healing efficiency of the composite. Tensile strength, as it is an important property in characterization of structural materials, is chosen as the self-healing parameter in this study in order to quantify the crack healing. In SFPMC, four different sets of specimens are tested in tension to demonstrate self-healing. They are designated as: Resin with undamaged fiber and catalyst (Virgin); Resin with undamaged fiber and no catalyst (Undamaged); Resin with damaged fiber and no catalyst (Damaged); and Resin with damaged fiber and catalyst that has healed (Healed)

Six samples of each specimen are tested in tension to predict how much of healing can be obtained in the composite. Tensile testing is performed according to ASTM D 30309/3039M, as the specimen is in rectangular shape. Loading rate during tensile testing is maintained at 2 $\mathrm{mm} / \mathrm{min}$ according to ASTM standard.

\section{Results and Discussion}

\subsection{Healing Process}

Fig. 2 demonstrates the schematics of the healing process. The figure outlines the general development of healing in a single fiber composite specimen where a crack initiated at the fiber is self-healed due to the timely reaction of the DCPD mixture (DCPD and chloroform) with Grubb's catalyst on the surface of the fiber.

When the hollow fiber breaks during damage initiation, the DCPD healing agent is observed seeping through the fiber in the area of the crack. The DCPD covers the surfaces of the crack in the matrix as well as the hollow fiber and the debonded region at the interface between the matrix and the fiber. Further observation after a few hours show that the area adjacent to the healed crack area within the hollow fiber still remains in a liquid form. In order to check localized healing, a single hollow fiber filled with healing agent and coated with catalyst is broken in the middle. There is an area that remained in a liquid form adjacent to the healed area demonstrating the attainment of localized healing/polymerization. Localized healing is always preferred as structures can be healed more than once in cases of repeated or multiple cracks using the remaining healing agent. 


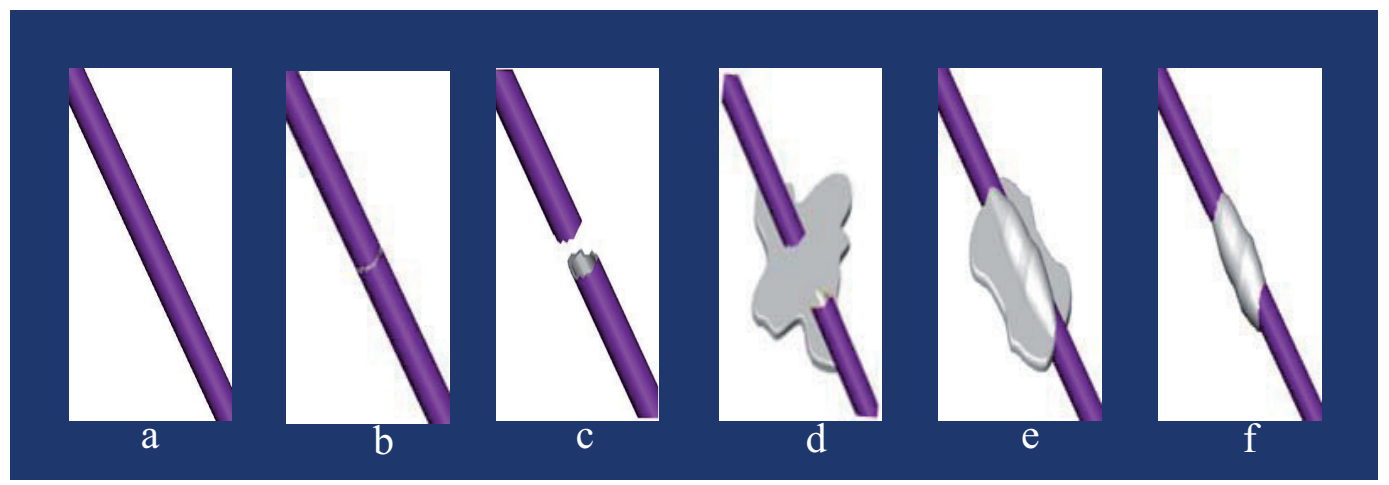

Figure 2. Schematic of the healing process - a close-up: (a) Unbroken/virgin fiber (b) Initial crack in the fiber (c) Fiber breaks (d) Healing agent oozing out of the fiber (e) Polymerization is initiated (f) Healing of the crack in the fiber and matrix.

The healing process is completed via ring-opening metathesis polymerization (ROMP) of DCPD monomer by the Grubbs' catalyst, as successfully used in microencapsulated composites [8]. The ring opening metathesis polymerization involves the use of Grubb's catalyst, a transition metal catalyst that has a high metathesis activity while being tolerant to a wide range of functional groups as well as water and oxygen [27].

\subsection{Tensile Tests}

Six replicates are tested in tension for each specimen type and the average of their tensile strength is obtained. The tensile tests are conducted after 12 hours of damage initiation. Previous works by Woldesenbet and Fikru have shown that minimum healing time is 12 hours [28]. It is observed during the test that, all samples broke into two sections with the fissure near or at the damage zone where healing has taken place. Healing efficiency, defined as the ratio of a material parameter of the healed to virgin materials. The material property can be the tensile or compressive ultimate strength, yield strength, shear strength, fracture strength, deflection, or modulus. Values of healing efficiency can therefore be obtained from experiments performed to determine the material properties mentioned. In this experiment, the tensile strength of the 'virgin' and 'healed' specimens is the material property investigated.

The tensile test results of single fiber polymer matrix composite (SFPMC) specimens are shown in Fig. 3. The experimental results show that a significant portion (90.7\%) of the tensile strength is restored due to the self-healing efficiency of the single fiber polymer matrix composite. The results from the tests carried out on SFPMC specimens confirm that self-healing is clearly achieved. The configurations of undamaged specimens with and without catalyst show similar results, $38.2 \mathrm{MPa}$ and $37.9 \mathrm{MPa}$ tensile strength, respectively. Both specimens have unbroken and undamaged fibers. The strength of the specimen with a catalyst is slightly higher, but the difference from the specimen without catalyst is within experimental error as indicated by the standard deviation. This result also proves that the catalyst does not have any deteriorating effect on the fiber-matrix interface.

The damaged specimen has achieved no healing since there are no catalysts and the tests are conducted without significant delay after damage has occurred. It has a broken fiber and adjacent damage zone. This specimen damaged by a flexural loading is equivalent to a single specimen with two equal size fibers extending from the middle to both ends. The damaged specimen is found to have a tensile strength of $26.9 \mathrm{MPa}$. This value is $30 \%$ lower than the strength of the virgin specimen. The remaining strength in the damaged specimen is obtained because of the matrix strength as well as due to the stress transfer between the matrix and the two broken parts of the fiber. The healed specimen has a tensile strength of $34.6 \mathrm{MPa}$ which is only $9.3 \%$ lower than the strength of the virgin specimen. 


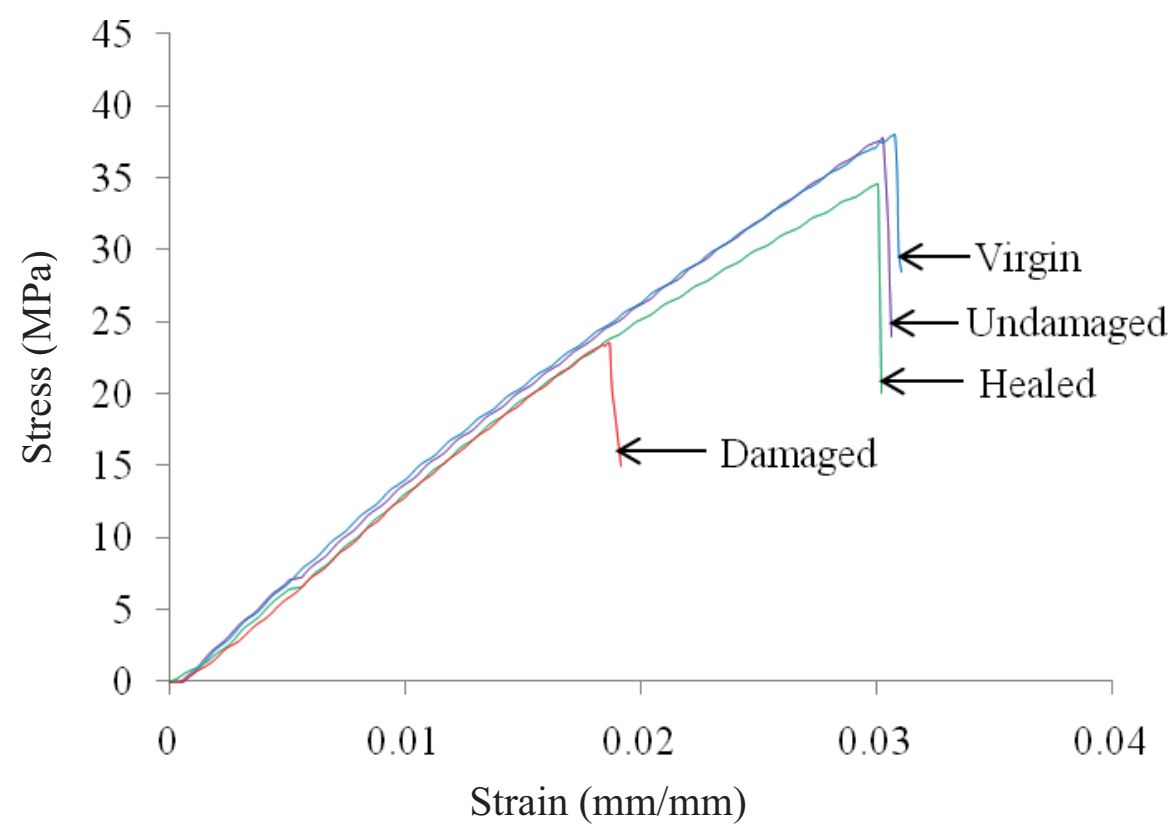

Figure 3. The stress versus strain diagram of the single damage SFPMC specimens.

The self-healing efficiency $(90.7 \%)$ obtained from the test is substantial; however the damage on the specimens is only at a single place. Multiple damages may occur on structures and therefore the self-healing efficiency has to be determined for cases when several cracks occur. In this research, the ability to achieve localized healing has enabled the possibility of conducting tests on specimens that have been damaged more than once. Thus a test is conducted on single fiber polymer composite specimens that are damaged at two places. A similar process of fabrication and testing is followed as in the case for a single damage specimen. However, there is no need to test undamaged samples because the previous (single damage) test has already confirmed that the Grubb's catalyst has no detrimental effect on the mechanical property of the composite.

The healed specimens that are broken at two locations (at one third of the fiber length from both ends) have recovered $80.4 \%$ of the virgin strength. In comparison with the result from the single damage test, the tensile strength of the multiple damages healed specimen is lower. This is expected as each damage does not completely $(100 \%)$ heal and therefore contributes to the larger reduction in strength for multiple damages. However, it should still be considered that significant self-healing has been achieved since the damaged specimen with no healing agent has only a tensile strength of $21.7 \mathrm{MPa}$, i.e., $56.8 \%$ of the virgin specimen's strength.

The tensile test results for the single fiber polymer matrix composites with the norboernene functionalized MWNT dispersed in the healing agent/DCPD mixture (SFPMN) show that a significant portion $(92.6 \%)$ of the tensile strength is restored due to the self-healing process. This result also shows that there is a direct relationship between the amount of MWNT and healing efficiency.

The single fiber polymer matrix composites results prove the feasibility of self-healing in fiber reinforced polymer matrix composites (FRPMC). The FRPMC test results are shown in Fig. 4. This experiment demonstrates that a considerable portion $(76.55 \%)$ of the tensile strength is restored due to the self-healing ability of the fiber reinforced polymer matrix composite. The standard deviation is calculated for the six samples in each type of specimen tested and is within $1 \%$ deviation from the average strength. The significant self-healing suggests that structures made of these materials are relatively damage tolerant that allow for more efficient use. 


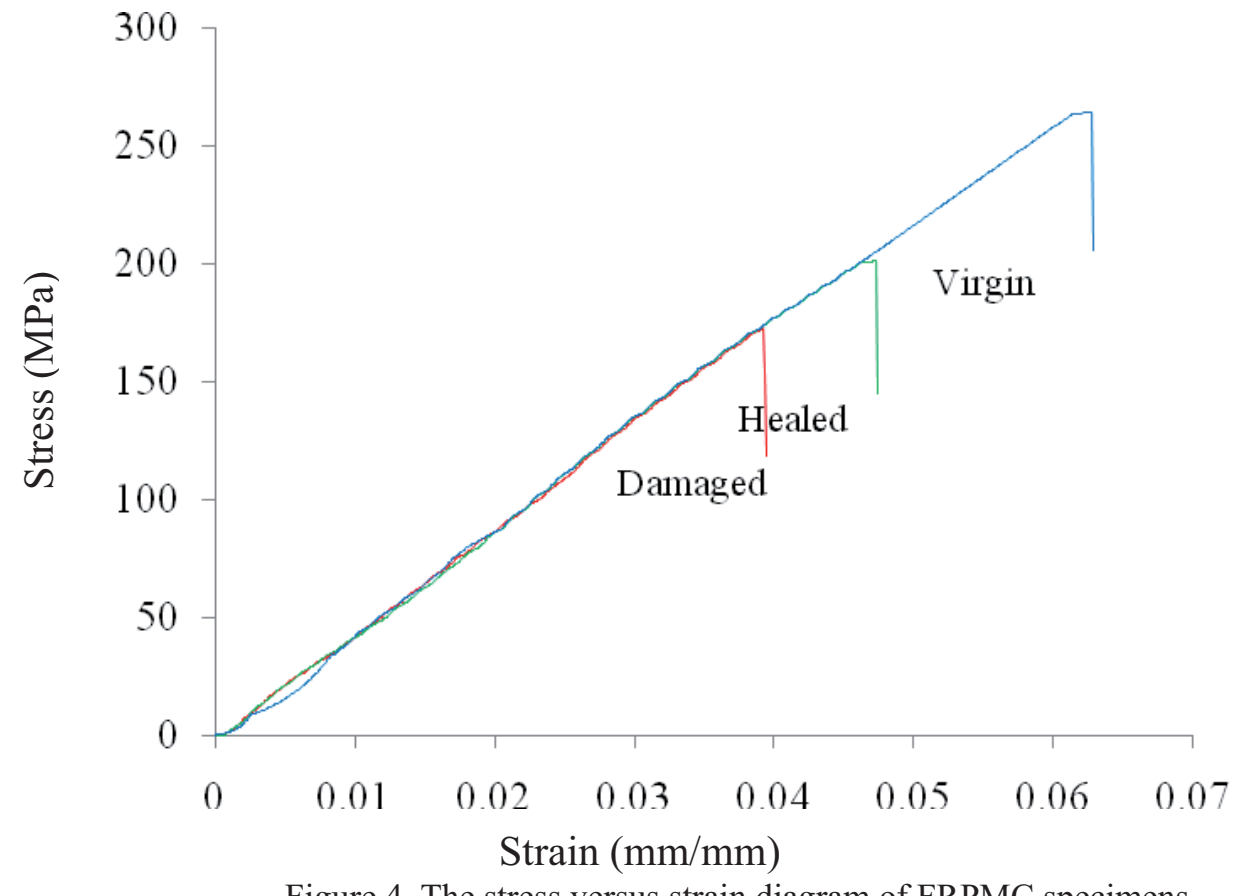

Figure 4. The stress versus strain diagram of FRPMC specimens.

\section{Conclusions}

This paper demonstrates self-healing in fiber reinforced polymer matrix composites. The study discusses three types of self-healing composite systems, single fiber polymer matrix composites (SFPMC), single fiber polymer matrix composites with functionalized multiwalled carbon nanotubes dispersed in the healing medium (SFPMN) and fiber reinforced polymer matrix composites (FRPMC). Tensile test results indicate that structures made out of these materials can retain significant portion of their tensile strength through self-healing.

The self-healing systems described in this paper include a DCPD healing agent, a Grubb's catalyst, hollow and solid fibers, and resin. In addition, the SFPMN specimens have norbornene functionalized multiwalled carbon nanotubes. The catalyst is placed on the outer surface of the hollow fiber to avoid untimely polymerization. Healing takes place after the healing agent polymerizes when it comes in contact with the Grubb's catalyst on the surface of the fiber during crack propagation. The catalyst is found to have no deteriorating effect on the fiber-matrix interface.

The SFPMC tests show that self-healing is achieved in a single fiber polymer matrix composite with $90.7 \%$ degree of restoration of the original tensile strength. Localized healing is achieved allowing the remaining healing agent to remain in a liquid form for further healing of cracks. When the SFPMC is subjected to damage at two places, a healing efficiency of $80.4 \%$ is obtained, showing that multiple damages as well can be healed significantly. In SFPMN, selfhealing is achieved in a single fiber test with $92.6 \%$ recovery of the original tensile strength. The addition of norbornene functionalized multiwalled carbon nanotubes has slightly enhanced the healing efficiency of composites compared to the healing agent system without the nanotubes. In FRPMC, the experiments show that self-healing is achieved with $76.55 \%$ degree of restoration of the original tensile strength of the composite with only $1.2 \%$ hollow fiber volume fraction. 


\section{Acknowledgements}

The authors would like to thank the support from the National Science Foundation, Grant Number 0600581.

\section{References}

1. Stavrinidis B, Holloway DG. Phys Chem Glasses 24:19-25 (1983).

2. Edvardsen C. ACI Materials Journa.l 96:448-454 (1999).

3. Wool RP, O'Conner KM. Journal of Applied Physics, 52:5953-5963 (1982).

4. Yu CC, Lin CB, Lee S. Journal of Applied Physics, 78:212-215 (1995).

5. Dry C. Composite Structures, 35:263-269 (1996).

6. Chen X, Wuld F, Mal AK, Shen H, Nutt SR. Macromolecules, 36:1802-1807 (2003).

7. Zako M, Takano N. Journal of Intelligent Material Systems and Structures, 10:836841 (1999).

8. White SR, Sottos NR, Geubelle PH, Moore JS, Kessler M R, Sriram SR, Brown EN, Viswanathan S. Nature, 409:794-797 (2001).

9. Brown EN, White SR, Sottos NR. Journal of Materials Science, 39:1703-1710 (2004).

10. Brown EN, White SR, Sottos NR.. Comp Sci Tech, 65(2):2474-2480 (2005).

11. Bleay SM, Loader CB, Hawyes VJ, Humberstone L, Curtis PT. Composites, 32(A):17671776 (2001).

12. Motuku M, Vaidya UK, Janowski GM. Smart Materials and Structures, 8:623-638 (1999).

13. Pang JWC, Bond IP. Composites, 36(A):183-188 (2005).

14. Pang JWC, Bond IP. Composites Science and Technology, 65:1791- 1799 (2005).

15. Trask RS, Bond IP. Smart Mater Struct, 15:704-710 (2006).

16. Kessler MR, Sottos NR, White SR. Composites, 34(A):743-753 (2003).

17. Toohey KS, Sottos NR, Lewis JA, Moore JS, White SR. Nature, 6:581-586 (2007).

18. Williams G, Trask RS, Bond IP. Composites Science and Technology, 68:3171-3177 (2008).

19. Williams G, Trask RS, Bond IP. Composites, 38(6):1525-1532 (2007).

20. Yin T, Rong MZ, Zhang MQ, Yang GC. Composite Science and Technology, 67:201-205 (2007).

21. Yin T, Zhou L, Rong MZ, Zhang MQ. Smart Materials and Structures, 17: 015-019 (2008).

22. Lee JY, Buxton GA, Balazs AC. J Chem Phys, 121:5531-1540 (2004).

23. Gutpa S, Zhang Q, Emrick T, Balazs AC, Russell TP. Nature Mater, 5:229-33 (2006).

24. Verberg R, Dale AT, Kumar P, Alexeev A, Balazs AC. J R Soc Interface, 4:349-357 (2007).

25. Jeong W, Kessler MR. Chemistry of Materials, 20:7060-7068 (2008).

26. Poche DS, Malter LM, Perrault JM. Polymer Bulletin, 43:43-49 (1999).

27. Grubbs RH, Tumas W. Science, 243:907-915 (1989).

28. Woldesenbet E, Fikru N. Proceedings of 23th Annual American Society of Composites Conference Proceedings. Memphis, Tennessee, USA. \#150, (2008). 